\title{
Delamination of laminate plate under tearing load mode
}

\author{
Eva Kormaníková ${ }^{1, *}$ and Kamila Kotrasová ${ }^{1}$ \\ ${ }^{1}$ Technical University of Košice, Faculty of Civil Engineering, Department of Structural Mechanics, \\ Vysokoškolská 4, 04200 Košice, Slovakia
}

\begin{abstract}
The paper presents the mixed mode delamination of laminate plate made of two sublaminates. To this purpose the load mode III of delamination is proposed as failure orthotropic model. Sublaminates are modeled by using shear deformable elements in program ANSYS. The response mixed-mode components of vector of energy release rates along the delamination front are calculated.
\end{abstract}

\section{Introduction}

The analysis of damage mechanisms in laminated composites has received a lot of interest for many years. Because of the low tensile strength in the transverse directions, interlaminar fracture represents a very important design consideration. As a matter of fact, because of their low interlaminar toughness, composite laminates often exhibit delamination when subjected to stress concentrations near manufacturing defects or to low velocity impacts. Delamination may arise at the edge of interlaminar defects in laminates loaded in compression, due to a global or local buckling of layers [1]. Moreover, delamination may occur as a failure mechanism in rehabilitated structures originating from interfacial defects between the repair composite laminate, made of fibre-reinforced plastic, and the existing concrete system [2].

A more efficient alternative for delamination analysis is to use plate theory. Classical delamination models [3], [4], [5] which consider the laminate as an assemblage of two plates in the cracked zone and of a unique plate in the undelaminated one, may give an accurate estimate of the total energy release rate $G$. Unfortunately, they are unable to accurately predict the individual energy release rates due to an inaccurate description of the local crack tip strain state and to take into account for shear deformations. As a consequence, the classical plate-based delamination models have been improved according to different methods. Delamination was modeled by adopting sublaminates governed by transverse shear deformable laminate theory, thus obtaining a reasonable approximation to the mode separation solution [6].

\footnotetext{
* Corresponding author: eva.kormanikova@tuke.sk
} 


\section{Mechanics of delaminated composite structure}

The mechanisms that lead to failure in composite materials are not yet fully understood, especially for matrix or fiber compression. Strength-based failure criteria are commonly used with the FEM to predict failure events in composite structures and different continuum-based criteria have been derived to relate the internal stresses and experimental measures of material strength to the onset of failure. In Fig. 1 a laminate contains a single in-plane delamination crack of area $\Omega_{D}$ with a smooth front $\partial \Omega_{D}$. The laminate thickness is denoted by $h_{0}$. The $x-y$ plane is taken to be the mid-plane of the laminate, and the z-axis is taken positive downwards from the mid-plane. For sublaminate modeling plate finite elements could be developed from shell elements. In this case each sublaminate is represented by an assemblage of the first order shear deformable (FSDT) plate elements bonded by zero-thickness interfaces in the transverse direction as shown in Fig. 1 [7]. Accordingly, the displacements in the $z$-th plate element, in terms of a global reference system located at the laminate mid-surface, are expressed [8]:

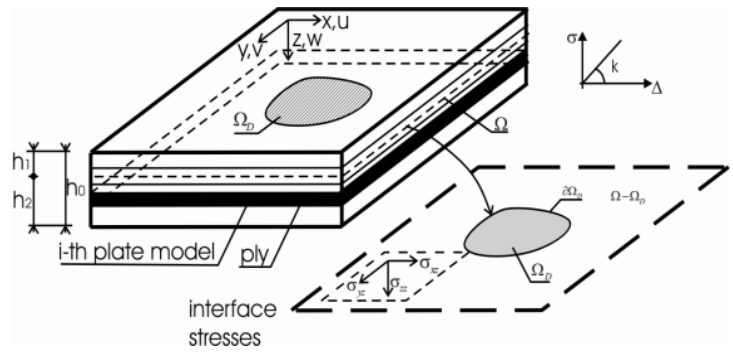

Fig. 1. Mechanics of the delaminated composite structure.

$$
\begin{aligned}
& U_{i}(x, y, z)=u_{i}(x, y)+\left(z-z_{i}\right) \psi_{x i}(x, y) \\
& V_{i}(x, y, z)=v_{i}(x, y)+\left(z-z_{i}\right) \psi_{y i}(x, y) \\
& W_{i}(x, y, z)=w_{i}(x, y)
\end{aligned}
$$

where $U_{i}, V_{i}$ refer to the in-plane displacements, and $W_{i}$ to the transverse displacements through the thickness of the $i$-th plate element, $u_{i}, v_{i}, w_{i}$ are the displacements at the midsurface of the $i$-th plate element, respectively, and $\psi_{x i}(x, y), \psi_{y i}(x, y)$ denote rotations of transverse normals about $y$ and $x$ axis, respectively.

At the reference surfaces, the membrane strain vector $\varepsilon_{i}$, the curvature $\kappa_{i}$, and transverse shear strain $\gamma_{i}$ respectively are defined as

$$
\left\{\begin{array}{l}
\varepsilon_{x i} \\
\varepsilon_{y i} \\
\gamma_{x y i}
\end{array}\right\}=\left\{\begin{array}{c}
\frac{\partial u_{i}}{\partial x} \\
\frac{\partial \mathrm{v}_{i}}{\partial y} \\
\frac{\partial u_{i}}{\partial y}+\frac{\partial \mathrm{v}_{i}}{\partial x}
\end{array}\right\},\left\{\begin{array}{c}
\kappa_{x i} \\
\kappa_{y i} \\
\kappa_{x y i}
\end{array}\right\}=\left\{\begin{array}{c}
\frac{\partial \psi_{x i}}{\partial x} \\
\frac{\partial \psi_{y i}}{\partial y} \\
\frac{\partial \psi_{x i}}{\partial y}+\frac{\partial \psi_{y i}}{\partial x}
\end{array}\right\},\left\{\begin{array}{l}
\gamma_{y z i} \\
\gamma_{x z i}
\end{array}\right\}=\left\{\begin{array}{c}
\psi_{x i}+\frac{\partial w_{i}}{\partial y} \\
\psi_{x i}+\frac{\partial w_{i}}{\partial x}
\end{array}\right\}
$$


By considering each plate as composed by one or several physical fiber-reinforced plies with their material axes arbitrarily oriented, the constitutive relations between stress resultants and corresponding strains are:

$$
\left\{\begin{array}{l}
\boldsymbol{N}_{i} \\
\boldsymbol{M}_{i}
\end{array}\right\}=\left[\begin{array}{ll}
\boldsymbol{A}_{i} & \boldsymbol{B}_{i} \\
\boldsymbol{B}_{i} & \boldsymbol{D}_{i}
\end{array}\right]\left\{\begin{array}{l}
\boldsymbol{\varepsilon}_{i} \\
\boldsymbol{\kappa}_{i}
\end{array}\right\}, \quad \boldsymbol{V}_{i}=\boldsymbol{H}_{i} \boldsymbol{\gamma}_{i}
$$

where $\boldsymbol{N}_{i}=\left\{N_{x i}, N_{y i}, N_{x y i}\right\}^{\mathrm{T}}$ is the membrane force resultant vector, $\boldsymbol{M}_{i}=\left\{M_{x i}, M_{y i}, M_{x y i}\right\}^{\mathrm{T}}$ the moment resultant vector and $\boldsymbol{V}_{i}=\left\{V_{y z i}, V_{x z i}\right\}^{\mathrm{T}}$ the transverse shear force resultant vector. In addition, $\boldsymbol{A}_{i}, \boldsymbol{D}_{i}, \boldsymbol{B}_{i}$ denote the classical extensional stiffness matrix, bending stiffness matrix and bending-extensional coupling stiffness matrix, respectively, whereas $\boldsymbol{H}_{i}$ is the shear stiffness matrix [8].

\section{Global and local approaches}

The stress singularities, captured by using the local approach, and stress resultant discontinuities, obtained in the context of the global approach, may be both responsible for energy release rates $G(s)$. Consequently they must represent the same mechanism of energy dissipation during delamination growth. The equivalence between local (interface) and global (plate) approaches can be proved by considering the regularized version of the modified total potential energy $\Pi_{r}$ and extracting discontinuities directly from the interface strain energy.

Using the interface constitutive equation $\{\sigma\}=[K]\{\Delta\}$ to compute interlaminar stresses, leads to the following total $G(s)$ expression

$$
G(s)=\lim _{k \rightarrow \infty} \frac{1}{2}\left[k_{z} \Delta w^{2}(s)+k_{x y} \Delta u^{2}(s)+k_{y x} \Delta v^{2}(s)\right]=G_{I}(s)+G_{I I}(s)+G_{I I I}(s), \Delta w(s) \geq 0
$$

where $s$ is a curvilinear coordinate along the delamination front. In (4) $G(s)$ is the local ERR function along the delamination front $\partial \Omega_{D}$, defined by

$$
-\int_{\partial \Omega_{D}} G(s) \dot{\Omega}_{D}(s) d s=\dot{\Pi}_{r}
$$

The mathematical correspondence between the local and global approaches can be thought as a direct consequence of the equivalence between the Lagrangian and the penalty methods to impose the adhesion constraint. These results show that two alternative methodologies leading to the same results can be used to compute the total energy release rate and its mode components: the one provided by the "strong" interface model", which imposes perfect adhesion ahead the delamination front in a limit process as the interface stiffness parameters approach infinity according to a penalty formulation; the other one based on the "collapsed" interface model in which adhesion is enforced directly by means of a Lagrangian multipliers formulation. However, the "strong" interface model leads to very simple formulas for both the total energy release rate and its individual mode components based on the limit values of interface strain energy densities which are very efficient when used in conjunction with the numerical finite element formulation. On the other hand, the "collapsed" interface model leads to more complex analytical formulas involving jumps in plate strains and stresses, which are necessary since it is not possible to retrieve interlaminar stresses and relative displacements between the surfaces of the delamination directly from the interface model. As a consequence, in the numerical calculations the "strong" interface model will be adopted. 


\section{Delaminated orthotropic plate}

A square plate comprising two subplates with thicknesses $h_{1}=0.5 \mathrm{~mm}, h_{2}=1 \mathrm{~mm}$ is investigated. The subplates are uniform loaded in tearing mode (Mode III) (Fig. 2, right). The plate geometry is shown in Fig. 2, left. The mechanical characteristics of the upper sublaminate (AS4D/9310) are: $E_{x}=134 \mathrm{GPa}, E_{y}=E_{z}=7.7 \mathrm{GPa}, G_{y z}=2.76 \mathrm{GPa}, G_{x y}=G_{x z}$ $=4.3 \mathrm{GPa}, v_{x y}=v_{x z}=0.3, v_{y z}=0.4$. The mechanical characteristics of the lower subplate (T300/5208) are: $E_{x}=136 \mathrm{GPa}, E_{y}=E_{z}=9.8 \mathrm{GPa}, G_{y z}=5.2 \mathrm{GPa}, G_{x y}=G_{x z}=4.7 \mathrm{GPa}, v_{x y}=$ $v_{x z}=0.28, v_{y z}=0.15$. The response mode components of energy release rate along the delamination front are calculated.

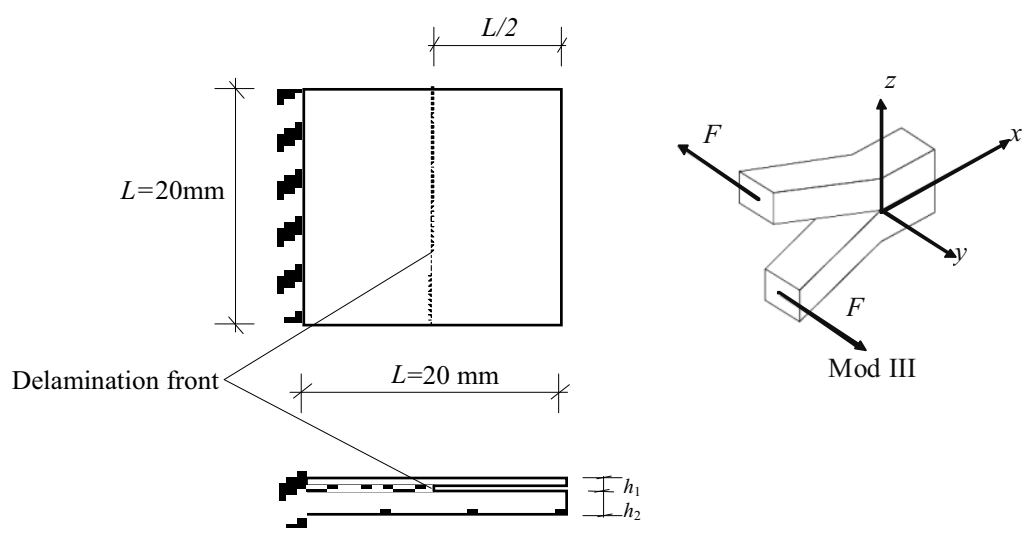

Fig. 2. A square plate geometry (left), tearing mode of delamination (right).
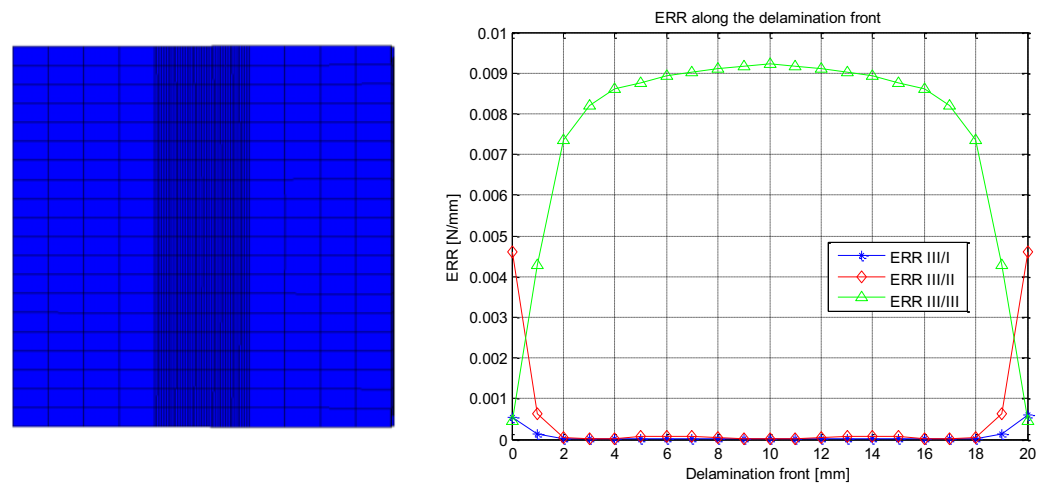

Fig. 3. Finite element model (left), results of individual components of ERR III (right).

Two plates have been used to model the delaminated plate in the thickness direction by using ANSYS. Each is modeled with SHELL181 elements. The projection on the $x-y$ plane of the finite element mesh, assumed equal for the plate and the interface models. The mesh is refined in a zone of $5 \times 20 \mathrm{~mm}^{2}$ centered with respect to the delamination front. In this zone, the length of the plate and interface elements in the $x$-direction is $0.125 \mathrm{~mm}$, whereas along the $y$-direction it is $1 \mathrm{~mm}$ (Fig. 3, left). A force of $1 \mathrm{~N} / \mathrm{mm}$ is imposed in the $y$-direction (tearing load mode III (Fig. 2)). Two subplates have been used to model the delaminated plate in the thickness direction. Interface elements, necessary to connect the two sublaminates, are implemented by using a combination of rigid links MPC 184 defined by two nodes and three degrees of freedom at each node. The delamination front was created by spring elements COMBIN14, in each offset node of the delamination front by 
three elements. The offset nodes can be generated by rigid links. The interface between subplates was modeled by constrain equations. When CE are used in place of rigid links, for each mid-plane node of the upper plate model, three coincident nodes located on the lower surface of the plate are created. Similarly, for each mid-plane node of the lower plate model, three coincident nodes located on the upper surface of the plate are created. Three COMBIN14 elements connected to the three pairs of coincident nodes placed at the delamination plane are introduced, each one acting in differ translation direction. For mixed-mode response conditions, a complete modeling of interface elements has been implemented. The stiffness of the spring elements binding the subplates is chosen as $10^{8}$ $\mathrm{N} / \mathrm{mm}^{3}$, that is appropriate for the normal and tangential stiffness.

\section{Conclusion}

A two-subplate orthotropic model [9] loaded by mode III was proposed as failure model. The material characteristics of the sublaminates were calculated by homogenization [10]. Subplates were modeled by using shear deformable elements of FEM [11] in program ANSYS. The individual components of ERR for loading mode III and response modes I, II and III, respectively along the delamination front are plotted in Fig. 3, right. Response mode III of ERR has maximum values in the middle of the model, therefore it is expected that the delamination proceeds from this place. Results have shown that response mode III is predominant and the response modes I and II are negligible, except of free edges. Based on these results a designer must pay attention at the free edges of delamination front [12$15]$.

This work was supported by the Scientific Grant Agency of the Ministry of Education of Slovak Republic and the Slovak Academy of Sciences under Projects VEGA 1/0477/15 and 1/0078/16.

\section{References}

1. D. Bruno, F. Greco, Int. J. Solids Struct. 37, 6239-6276 (2000)

2. Y. M. Lim, V. C. Li, Cement Concr. Compos. 19, 373-385 (1997)

3. B. Cochelin, M. Potier-Ferry, Comput. Methods, Appl. Mech. 89, 361-380 (1991)

4. J. - K. Kim, Comput. Structures 26, 6, 975-983 (1997)

5. D. Bruno, F. Greco, Int. J. Solids Struct. 38/50-51, 9149-9177 (2001)

6. Z. Zou, S. R. Reid, P. D. Soden, S. Li, J. Solids Structures 38, 2597-2613 (2001)

7. M. Zmindak, V. Dekys, P. Novak, Adv. Mat. Res. 969, 176-181, (2014)

8. E. J. Barbero, Finite Element Analysis of Composite Materials (CRC Press, Boca Raton, 2008)

9. E. J. Barbero, Introduction to Composite Materials Design (Taylor and Francis, NY., 1999)

10. M. Sejnoha, L. Kucikova, V. Kralik, J. Sejnoha, Civil-Comp Proceedings 108, (2015)

11. V. Michalcova, L. Lausova, Civil-Comp Proceedings, 108, (2015)|

12. M. Krejsa, L. Koubova, J. Flodr, J. Protivinsky, Q. T. Nguyen, Frattura ed Integrita Strutturale 11(39), 143-159, (2017)

13. M. Mihalikova, M. Nemet, M. Vojtko, Key Eng. Mat. 635, 118-121, (2015)

14. J. Melcer, G. Lajcakova, Adv. Mat. Res. 969, 85-88, (2014)

15. E. Kormanikova, K. Kotrasova, Key Eng. Mat. 635, 85-88, (2015) 